\title{
Constitutive activation of p70 S6 kinase is associated with intrinsic resistance to cisplatin
}

\author{
ROHINI DHAR and ALAKANANDA BASU \\ Department of Molecular Biology and Immunology, University of North Texas Health \\ Science Center and Institute for Cancer Research, Fort Worth, TX 76107, USA
}

Received December 10, 2007; Accepted February 15, 2008

\begin{abstract}
Cisplatin is widely used for the treatment of solid tumors, including small cell lung cancers, but its success is often compromised due to relapse and resistance to further treatment. p70 ribosomal S6 kinase (p70S6K) has been shown to be upregulated in lung cancer cells. In the present study, we investigated whether the p70S6K pathway contributes to cisplatin resistance in human small cell lung cancer H69 cells. The levels of phosphorylated p70S6K and its downstream target S6 but not total p70S6K or S6 were elevated in the $\mathrm{H} 69$ cells that acquired resistance to cisplatin (H69/CP) compared to parental H69 cells. Cisplatin treatment resulted in the activation of p70S6K and downregulation of p70S6K was associated with cisplatin-induced PARP cleavage. While the ability of cisplatin to induce apoptosis was attenuated in H69/CP cells, inhibition of p70S6K by rapamycin enhanced cisplatin-induced apoptosis in these cells as evident by the increase in cisplatin-induced poly(ADP-ribose) polymerase (PARP) cleavage. The phosphoinositide 3-kinase (PI3K) inhibitor Ly294002 alone induced PARP cleavage and further augmented cisplatin-induced PARP cleavage. In contrast, inhibition of extracellular signal-regulated kinase (ERK) by U0126 attenuated cisplatin-induced PARP cleavage. Both rapamycin and Ly294002 enhanced cisplatin-induced activation of ERK1/2. Taken together, these results suggest that activation of p70S6K contributes to cisplatin resistance in small cell lung cancer H69 cells, and inhibition/downregulation of p70S6K as well as activation of ERK1/2 could circumvent cisplatin resistance.
\end{abstract}

\section{Introduction}

Cisplatin is widely used for the therapy of several types of cancer, including testicular, ovarian, cervical, and small cell

Correspondence to: Dr Alakananda Basu, Department of Molecular Biology and Immunology, University of North Texas Health Science Center, Fort Worth, TX 76107, USA

E-mail: abasu@hsc.unt.edu

Key words: p70S6K, cisplatin, apoptosis, small cell lung cancer lung cancer (SCLC) (1-3). Cisplatin treatment is, however, limited due to the acquisition of resistance by tumor cells to the drug and its toxic side effects (4-6). The mechanism(s) of cisplatin resistance is often multifactorial and an understanding of cisplatin resistance is critical to exploit this drug effectively for the treatment of cancers.

p70S6K is a serine/threonine protein kinase responsible for the phosphorylation and activation of 40S ribosomal subunit protein S6 $(7,8)$. It acts downstream of the mammalian target of rapamycin (mTOR). The activation of p70S6K and its downstream target ribosomal protein S6 mediates nutrient and mitogen-stimulated translation, which is essential for cell growth and proliferation (9). Activation of S6 results in the translation of a class of mRNA which contains an oligopyrimidine tract at their transcriptional start site. This class of mRNAs encodes for many of the components of the protein synthesis apparatus. Due to its involvement in regulating translation, cell cycle progression, differentiation and cell motility, p70S6K has been implicated as an oncogene (10).

A number of studies have focused on the involvement of p70S6K in influencing cellular responses to apoptotic stimuli. Treatment of Swiss 3T3 and RAT-1 cells with etoposide and staurosporine resulted in dephosphorylation and decreased activity of p70S6K $(11,12)$. It has also been shown that cisplatin inhibited the phosphorylation of p70S6K in mouse myoblasts (13). Rapamycin, an immunosuppressant blocked the activation of $\mathrm{mTOR} / \mathrm{p} 70 \mathrm{~S} 6 \mathrm{~K}$, thus compromising the cell's ability to progress through the $\mathrm{G} 1$ phase of the cell cycle $(14,15)$. Rapamycin derivatives are in clinical trials for the treatment of several cancer types (16) although the induction of apoptosis by rapamycin is not universal $(17,18)$. It has been reported that rapamycin can synergize with other agents such as tamoxifen, imanitib and doxorubicin to enhance apoptosis (19-22). Similarly, rapamycin derivatives enhanced cisplatin-induced cell death in ovarian, leukemia and lung cancers (23-26). In contrast, it has been reported that rapamycin did not induce apoptosis in Rat-1 and PC12 cells and thymocytes $(17,18)$.

Cisplatin is often used for the treatment of human SCLCs yet acquisition of resistance by these tumors to cisplatin is a major problem in the therapy of SCLC (27). It has been reported that p70S6K pathway is constitutively activated in several SCLCs (10) and activation of p70S6K was associated with anchorage-independent growth of SCLC (28). In addition, rapamycin analog CCI779 restored cisplatin sensitivity in 
cisplatin-resistant small cell lung cancer cells $(26,29)$ although it is not known if p70S6K signaling pathway is affected when cells acquired resistance to cisplatin. Recently, it has been reported that cisplatin resistance of non-small cell lung cancer A549 cells was associated with amplification of Aktl gene, resulting in activation of its downstream target mTOR pathway (30). In the present study, we investigated if p70S6K signaling pathway is compromised when SCLC H69 cells acquire resistance to cisplatin. We have demonstrated that p70S6K is constitutively activated in SCLC H69 cells that acquired resistance to cisplatin, and inhibition of p70S6K enhanced cellular sensitivity to cisplatin. Furthermore, inhibition of PI3K/Akt and mTOR pathway resulted in activation of ERK and reversed cisplatin resistance.

\section{Materials and methods}

Materials. Polyclonal antibodies to p70S6K, phospho-p70S6K (T389), Akt, phospho-Akt (S473), S6 and phospho-S6 (S235/236), were purchased from Cell Signaling Technology, Inc. (Danvers, MA). Monoclonal antibodies to GAPDH and phospho-ERK1/2 were purchased from Santa Cruz Biotechnology, Inc. (Santa Cruz, CA). Monoclonal antibody to PARP was purchased from Pharmingen (San Diego, CA) and ERK1/2 antibody was purchased from BD Biosciences. Monoclonal antibody to actin was obtained from Amersham (Arlington Heights, IL). Horseradish peroxidase-conjugated goat anti-mouse and donkey anti-rabbit antibodies were obtained from Jackson ImmunoResearch (West Grove, PA). The enhanced chemiluminescence detection kit was from Amersham (Piscataway, NJ).

Cell culture. Parental SCLC H69 cells and cells selected for resistance to cisplatin $(\mathrm{H} 69 / \mathrm{CP})$ were generously provided by Dr Nagahiro Saijo (National Cancer Center Research Institute, Tokyo, Japan). Cells were maintained in RPMI-1640 (Life Technologies Inc., Grand Island, NY) supplemented with $2 \mathrm{mM}$ glutamine and $10 \%(\mathrm{v} / \mathrm{v})$ heat-inactivated fetal bovine serum at $37^{\circ} \mathrm{C}$ in the presence of $5 \% \mathrm{CO}_{2}$.

Immunoblot analysis. Cells were lysed in M-PER mammalian extraction buffer (Pierce, Rockford, IL) containing $1 \mathrm{mM}$ DTT and protease inhibitors. Equal amounts of total protein were separated by $10 \%(\mathrm{w} / \mathrm{v})$ SDS-PAGE and transferred onto a poly(vinylidene difluoride) membrane. Western blot analyses were performed as described before (10). The blot was probed with antibody to either GAPDH or actin to control for equal loading.

\section{Results}

Comparison of p70S6K status in parental and cisplatinresistant human small cell lung cancer (SCLC) H69 cells. To determine if the p70S6K pathway is affected when human H69 SCLC cells acquire resistance to cisplatin, we compared the level and activation status of p70S6K in parental H69 cells and its cisplatin-resistant variant H6/CP cells. Fig. 1A shows that there was little change in the total level of p70S6K in the cisplatin-resistant $\mathrm{H} 69 / \mathrm{CP}$ cells as compared to the parental H69 cells. Phosphorylation of p70S6K at threonine

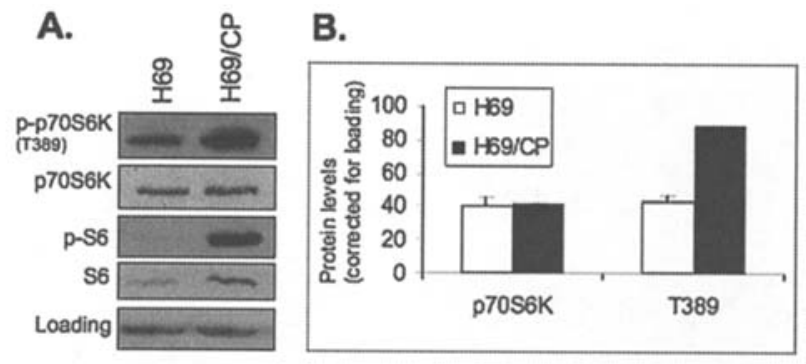

Figure 1. Comparison of p70S6K status in parental and cisplatin-resistant human small cell lung cancer (SCLC) H69. A, Western blot analysis was performed with total cellular extracts using indicated antibodies. GAPDH was used as a loading control. Results are representative of three independent experiments. B, Densitometric quantification of total and phospho-p70S6K corrected for loading. Data are mean \pm SEM of three independent experiments.

389 (T389) is important for the activation of p70S6K $(31,32)$. Therefore, we compared the phosphorylation status of p70S6K at T389 in the H69 and H69/CP cells. Phosphorylation of p70S6K at T389 was increased by approximately 2-fold in H69/CP cells compared to H69 cells. In addition, the level of phosphorylated S6 (p-S6), which is a downstream target of p70S6K, was also much higher in the H69/CP cells compared to parental cells, indicating increased activation of p70S6K in the H69/CP cells. These results suggest that cisplatin resistance was associated with an increase in phosphorylation/activation of $\mathrm{p} 70 \mathrm{~S} 6 \mathrm{~K}$.

Effect of cisplatin on p70S6K activation and PARP cleavage in H69 and H69/CP cells. Since the level of phosphorylated p70S6K was constitutively higher in the cisplatin-resistant cells, we compared the effect of cisplatin on p70S6K phosphorylation and cell death in H69 and H69/CP cells. Activation of caspase-3 or -7 during apoptosis cleaves full-length $115-\mathrm{kDa}$ poly(ADP-ribose) polymerase (PARP) to an $85-\mathrm{kDa}$ fragment and cleavage of PARP is used as a measure of apoptosis (33). Fig. 2 shows that a 48 -h exposure to cisplatin caused a concentration-dependent increase in PARP cleavage in H69 cells such that the cleaved fragment of PARP appeared when cells were treated with $20 \mu \mathrm{M}$ cisplatin and increased further when treated with $40 \mu \mathrm{M}$ cisplatin. In contrast, $40 \mu \mathrm{M}$ cisplatin had little effect on the cleavage of PARP in H69/CP cells. Cisplatin caused a concentration-dependent increase in the phosphorylation of p70S6K in both H69 and H69/CP cells. However, while increase in p70S6K phosphorylation was apparent when H69/CP cells were treated with $10 \mu \mathrm{M}$ cisplatin, higher concentrations of cisplatin were required to induce p70S6K phosphorylation in $\mathrm{H} 69$ cells. The level of p70S6K also decreased when H69 cells but not H69/CP cells were treated with $40 \mu \mathrm{M}$ cisplatin, suggesting that decrease in p70S6K protein level correlates with cisplatin-induced cell death.

Comparison of the effect of rapamycin on cisplatin-induced cell death in $\mathrm{H69}$ and H69/CP cells. Rapamycin is a macrolide fungicide that possesses potent antimicrobial, immunosuppressant, and antitumor properties (16). Rapamycin leads to the dephosphorylation and inactivation of p70S6K (34). 


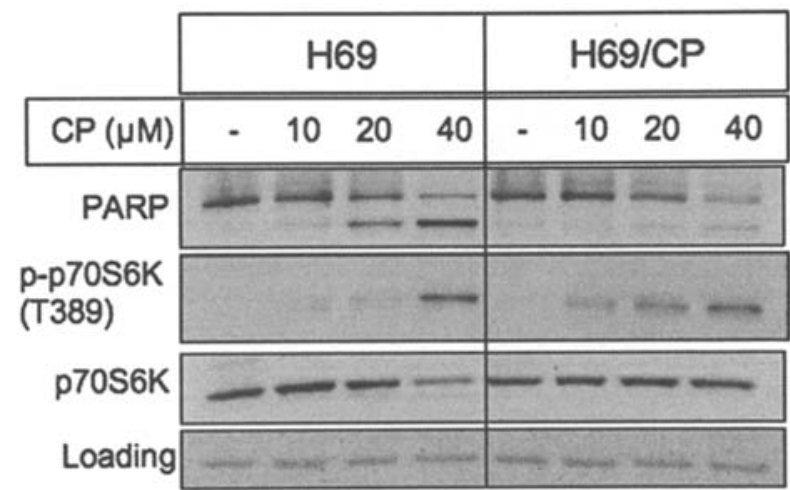

Figure 2. Effect of cisplatin on p70S6K activation and PARP cleavage in H69 and H69/CP cells. H69 and H69/CP cells were treated with indicated concentrations of cisplatin for $48 \mathrm{~h}$. Western blot analysis was performed with total cellular extracts using indicated antibodies. GAPDH was used as a loading control. Results are representative of three independent experiments.

A.

\begin{tabular}{|c|c|c|}
\hline & H69 & $\mathrm{H} 69 / \mathrm{CP}$ \\
\hline $\mathrm{CP}(\mu \mathrm{M})$ & $\begin{array}{lll}- & 10 & 25\end{array}$ & $\begin{array}{lll}- & 10 & 25\end{array}$ \\
\hline $\operatorname{Rap}(\mathrm{nM})$ & -+-+-+ & -+-+-+ \\
\hline PARP & $\lim _{-\infty}-\infty$ & $\sin _{-\infty}$ \\
\hline $\begin{array}{l}\text { p-p70S6K } \\
\text { (т389) }\end{array}$ & & 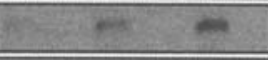 \\
\hline p70S6K & $-\infty-\infty-1$ & ------ \\
\hline Loading & $\infty$ & -10 \\
\hline
\end{tabular}

B.

\begin{tabular}{|c|c|c|}
\hline & $\mathrm{H} 69$ & $\mathrm{H} 69 / \mathrm{CP}$ \\
\hline $\mathrm{CP}(\mu \mathrm{M})$ & $\begin{array}{ll}- & 10 \quad 25\end{array}$ & $\begin{array}{lll}- & 10 & 25\end{array}$ \\
\hline $\operatorname{Rap}(\mathrm{nM})$ & -+-+-+ & $-+-+\cdot+$ \\
\hline $\mathrm{p}-\mathrm{S} 6$ & $-5-10$ & 625 \\
\hline s6 & betabe & 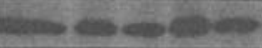 \\
\hline Loading & 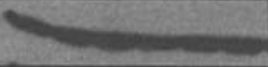 & 10 \\
\hline
\end{tabular}

Figure 3. Comparison of the effect of rapamycin on cisplatin-induced cell death in H69 and H69/CP cells. A and B, H69 and H69/CP cells were treated with $20 \mathrm{nM}$ rapamycin for 30 min prior to treatment with 10 and $25 \mu \mathrm{M} \mathrm{CP}$ for $48 \mathrm{~h}$. Western blot analysis was performed with total cellular extracts using indicated antibodies. Actin was used as a loading control. Results are representative of two independent experiments.

Fig. 3A shows that rapamycin alone had little effect on PARP cleavage in either H69 or H69/CP cells. While rapamycin enhanced cisplatin-induced PARP cleavage when H69 cells were treated with $10 \mu \mathrm{M}$ cisplatin, rapamycin had no additional effect when H69 cells were treated with $25 \mu \mathrm{M}$ cisplatin.
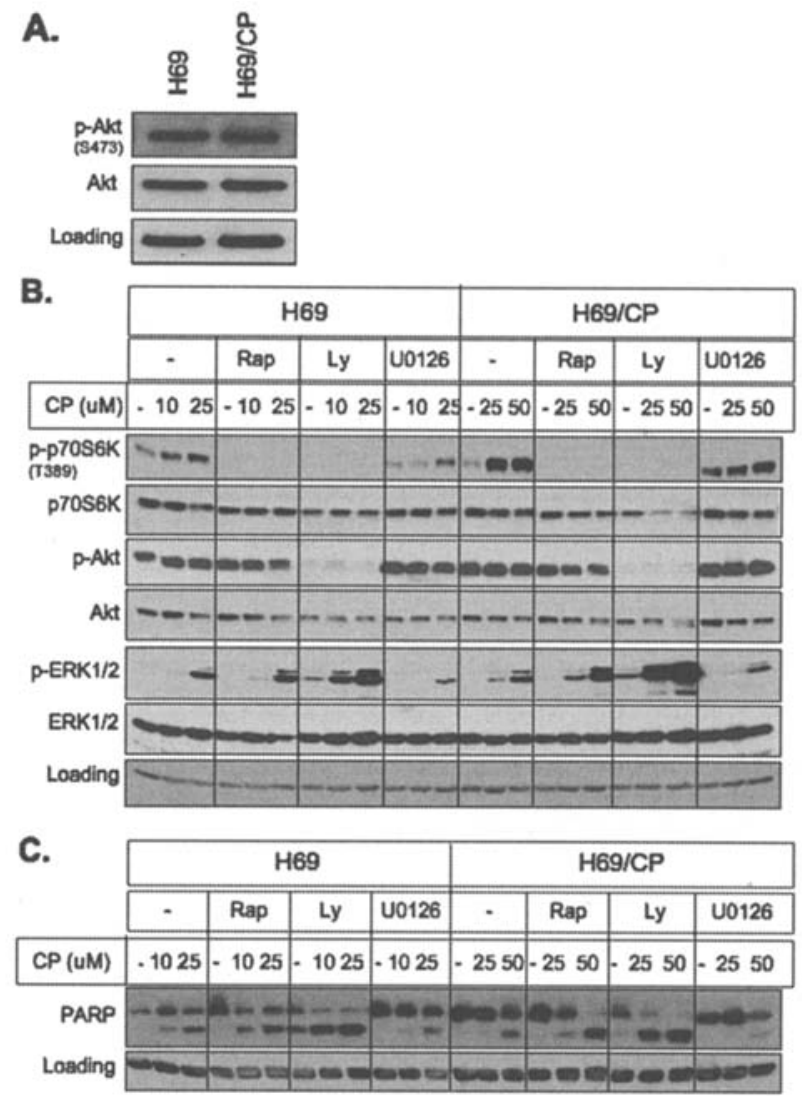

Figure 4. The effect of PI3K inhibitor LY294002 on p70S6K phosphorylation and cisplatin-induced cell death. A, Western blot analysis was performed with total cellular proteins using indicated antibodies. GAPDH was used as a loading control. B and C, H69 and H69/CP cells were treated with $20 \mathrm{nM}$ rapamycin, $25 \mu \mathrm{M} \mathrm{LY} 294002$ and $10 \mu \mathrm{M} \mathrm{U} 0126$ for $30 \mathrm{~min}$ prior to treatment with indicated concentrations of cisplatin for $48 \mathrm{~h}$. Western blot analysis was performed with total cellular extracts using indicated antibodies.

Treatment of H69/CP cells with either rapamycin or cisplatin alone had little effect on PARP cleavage. However, pretreatment with rapamycin enhanced sensitivity of $\mathrm{H} 69 / \mathrm{CP}$ cells to cisplatin considerably such that in the presence of rapamycin $25 \mu \mathrm{M}$ cisplatin was equally effective in inducing PARP cleavage in both $\mathrm{H} 69$ and H69/CP cells. Cisplatin caused a concentration-dependent increase in phosphorylation of p70S6K at T389 site and treatment with rapamycin inhibited phosphorylation of p70S6K at T389. Cisplatin-induced phosphorylation of p70S6K correlated with phosphorylation of S6, a downstream target of p70S6K (Fig. 3B), suggesting that $\mathrm{p} 70 \mathrm{~S} 6 \mathrm{~K}$ is activated by cisplatin treatment. As expected, rapamycin inhibited phosphorylation of S6. Thus, dephosphorylation of p70S6K by rapamycin may be associated with increase in cisplatin-mediated cell death.

The effect of PI3K inhibitor Ly294002 on p70S6K phosphorylation and cisplatin-induced cell death. There are controversies whether or not PI3K/Akt signaling pathway acts upstream of mTOR (35-37). In addition, it has recently been reported that amplification of Aktl gene in cisplatin-resistant non-small cell lung cancer A549 cells could result in activation of p70S6K (30). We therefore compared the levels of total 
and phospho-Akt in $\mathrm{H} 69$ and H69/CP cells. Fig. 4A indicates that levels of total and phosphorylated Akt were comparable in $\mathrm{H} 69$ and H69/CP cells. We also examined the effect of Ly294002, an inhibitor of PI3K/Akt, on p70S6K phosphorylation and cisplatin-induced cell death. Fig. 4B shows that both $\mathrm{H} 69$ and H69/CP cells contained high level of phospho-Akt and Ly294002 but not rapamycin inhibited phosphorylation of Akt in these cells. However, Ly294002 was as effective as rapamycin in inhibiting phosphorylation of p70S6K, suggesting that PI3K/Akt acts upstream of mTOR/ p70S6K in these cells. Since Akt may act upstream of mitogenactivated protein kinase/extracellular signal-regulated kinase kinase (MEK/ERK) signaling pathway which may also regulate p70S6K (38), we examined the effect of MEK inhibitor U0126 on the phosphorylation of ERK1/2, Akt and p70S6K. As shown in Fig. 4B, U0126 attenuated cisplatin-induced activation of ERK but not Akt. It also caused a modest decrease in p70S6K phosphorylation. Interestingly, both rapamycin and Ly294002 enhanced cisplatin-induced activation of ERK although Ly294002 was much more effective compared to rapamycin. We also compared the effects of these inhibitors on cisplatin-induced cell death. As shown in Fig. 4C, Ly294002 alone induced PARP cleavage and it caused substantial increase in cisplatin-induced PARP cleavage. In contrast, U0126 inhibited cisplatin-induced apoptosis in both H69 and H69/CP cells. There was a good correlation between activation of ERK (Fig. 4B) and cleavage of PARP (Fig. 4C). These results suggest that activation of ERK was associated with cisplatin-induced cell death.

\section{Discussion}

The efficacy of cisplatin not only depends on its ability to induce DNA damage but also on the cells' ability to respond to this damage. The crucial point in these events may be determined by signaling events downstream of the drugDNA interactions. Acquisition of resistance by tumor cells to anticancer drugs may involve modifications in the cells' ability to trigger downstream signaling events. It is thus the balance between the various proteins present in the cell that finally decides whether the cell should live or die. Although the involvement of p70S6K in DNA damage-induced cell death is established (11-13), little is known about how p70S6K is affected when cells acquire resistance to cisplatin. The results of our present study revealed that an increase in the levels of active p70S6K was associated with cisplatin resistance in human small cell lung cancer H69 cells. In addition, inhibition of p70S6K phosphorylation by rapamycin or Ly294002 could restore cisplatin sensitivity in cisplatinresistant H69 cells. Taken together, these results suggest that treatment with cisplatin during the selection process caused a modification in the p70S6K signaling pathway.

We have found that cisplatin caused activation of p70S6K in both H69 and H69/CP cells as determined by its phosphorylation at T389 site and its downstream target S6 although the extent of p70S6K activation was greater in H69/CP cells compared to $\mathrm{H} 69$ cells. This is consistent with a recent report that tumor necrosis factor- $\alpha$ activated the phosphorylation of p70S6K in MCF-7 cells (39). However, this is in contrast to a previous report that showed that cisplatin had little effect on
p70S6K phosphorylation in cisplatin resistant SR2 cells whereas it in fact decreased p70S6K phosphorylation in the sensitive cell line (26). This apparent anomaly could be explained by the fact that growth factors present in the serum could affect p70S6K phosphorylation. We have found that when cells were treated with cisplatin in fresh FBS containing media, the constitutive p70S6K phosphorylation was much higher (data not shown). However, when cells were incubated with different concentrations of cisplatin for $48 \mathrm{~h}$, the basal p70S6K phosphorylation was low and cisplatin caused an increase in p70S6K phosphorylation. In addition, we found that higher concentrations of cisplatin that resulted in cell death also induced downregulation of total p70S6K. Since cisplatin-resistant cells require higher concentrations of cisplatin to induce cell death, the decrease in total as well as phospho-p70S6K is less in the resistant cells compared to drug-sensitive parental cells.

p70S6K is believed to act downstream of PI3K/Akt $(35,40)$. A recent report attributed amplification of Aktl gene to be the cause of p70S6K activation and cisplatin resistance in A549 non-small cell lung cancer cells (30). On the contrary, we found that there was no difference in the levels of constitutive and/or phosphorylated Akt in H69 and H69/CP cells. Furthermore, cisplatin had little effect on Akt phosphorylation in either cell type. We have also found that inhibition of Akt by Ly294002 caused substantial increase in cisplatin-induced cell death. In fact, Ly294002 alone caused modest cell death, suggesting the importance of Akt signaling pathway in the survival of these SCLC cells. However, the effect of Ly294002 was much greater compared to rapamycin, suggesting that Ly294002 may act via additional pathway besides inhibiting p70S6K.

Since Akt signaling pathway can also affect the mitogenactivated protein kinase (MAPK) pathway (41) we compared the effect of Ly294002 with the MEK inhibitor U0126. We have found that in contrast to Ly294002, U0126 attenuated cisplatin-induced cell death in both parental and cisplatinresistant $\mathrm{H} 69$ cells. In addition, cisplatin caused activation of ERK1/2. This is consistent with our previous report that activation of ERK1/2 was associated with cellular sensitivity to cisplatin (42). In this report we found that Ly294002 caused an increase in both basal and cisplatin-induced ERK1/2 phosphorylation. In fact, the extent of ERK $1 / 2$ phosphorylation correlated with the extent of PARP cleavage. Thus, Ly294002 not only inhibits the Akt/mTOR/p70S6K pathway, it also activates the MEK/ERK pathway. Rapamycin also caused a modest increase in cisplatininduced activation of ERK1/2. Thus, activation of ERK may also contribute to cisplatin sensitization by these inhibitors. With lung cancer accounting for the largest number of cancer deaths and acquisition of resistance resulting in poor prognosis, treatment with a combination of drugs may restore cisplatin sensitivity.

\section{Acknowledgments}

The authors thank Dr Nagahiro Saijo for providing us H69 cells. This work was supported by the grants CA85682 and CA71727 from the National Cancer Institute. Rohini Dhar is supported by the NSF Score grant 0440334. 


\section{References}

1. Radulovic S, Tesic Z and Manic S: Trans-platinum complexes as anticancer drugs: recent developments and future prospects. Curr Med Chem 9: 1611-1618, 2002.

2. Seki K, Yoshikawa H, Shiiki K, Hamada Y, Akamatsu N and Tasaka K: Cisplatin (CDDP) specifically induces apoptosis via sequential activation of caspase- $8,-3$ and -6 in osteosarcoma. Cancer Chemother Pharmacol 45: 199-206, 2000.

3. Boulikas T and Vougiouka M: Recent clinical trials using cisplatin, carboplatin and their combination chemotherapy drugs (review). Oncol Rep 11: 559-595, 2004.

4. Marzano C, Bettio F, Baccichetti F, Trevisan A, Giovagnini L and Fregona D: Antitumor activity of a new platinum(II) complex with low nephrotoxicity and genotoxicity. Chemico-Biol Interact 148: 37-48, 2004.

5. Bianchi R, Gilardini A, Rodriguez-Menendez V, et al: Cisplatininduced peripheral neuropathy: neuroprotection by erythropoietin without affecting tumour growth. Eur J Cancer 43: 710-717, 2007.

6. Rabik CA and Dolan ME: Molecular mechanisms of resistance and toxicity associated with platinating agents. Cancer Treat Rev 33: 9-23, 2007.

7. Yoon P, Giafis N, Smith J, et al: Activation of mammalian target of rapamycin and the p70 S6 kinase by arsenic trioxide in BCR-ABL-expressing cells. Mol Cancer Ther 5: 2815-2823, 2006.

8. Jefferies HBJ, Reinhard C, Kozma SC and Thomas G: Rapamycin selectively represses translation of the 'polypyrimidine tract' mRNA family. Proc Natl Acad Sci USA 91: 4441-4445, 1994.

9. Pullen N and Thomas G: The modular phosphorylation and activation of p70s6k. FEBS Lett 410: 78-82, 1997.

10. Seufferlein T and Rozengurt E: Rapamycin inhibits constitutive p70s6k phosphorylation, cell proliferation, and colony formation in small cell lung cancer cells. Cancer Res 56: 3895-3897, 1996.

11. Tee AR and Proud CG: DNA-damaging agents cause inactivation of translational regulators linked to mTOR signalling. Oncogene 19: 3021-3031, 2000.

12. Tee AR and Proud CG: Staurosporine inhibits phosphorylation of translational regulators linked to mTOR. Cell Death Differ 8: 841-849, 2001

13. Wan X and Helman LJ: Effect of insulin-like growth factor II on protecting myoblast cells against cisplatin-induced apoptosis through p70 S6 kinase pathway. Neoplasia 4: 400-408, 2002.

14. Price DJ, Grove JR, Calvo V, Avruch J and Bierer BE: Rapamycin-induced inhibition of the 70-kilodalton $\mathrm{S} 6$ protein kinase. Science 257: 973-977, 1992.

15. Lane HA, Fernandez A, Lamb NJC and Thomas G: p70s6k function is essential for G1 progression. Nature 363: 170-172, 1993.

16. Wan X and Helman LJ: The biology behind mTOR inhibition in Sarcoma. Oncologist 12: 1007-1018, 2007.

17. Yao R and Cooper GM: Growth factor-dependent survival of rodent fibroblasts requires phosphatidylinositol 3-kinase but is independent of pp70S6K activity. Oncogene 13: 343-351, 1996.

18. McCarthy SA, Cacchione RN, Mainwaring MS and Cairns JS: The effects of immunosuppressive drugs on the regulation of activation-induced apoptotic cell death in thymocytes. Transplantation 54: 543-547, 1992.

19. DeGraffenried LA, Friedrichs WE, Russell DH, et al: Inhibition of mTOR activity restores tamoxifen response in breast cancer cells with aberrant akt activity. Clin Cancer Res 10: 8059-8067, 2004.

20. Burchert A, Wang Y, Cai D, et al: Compensatory PI3-kinase/ $\mathrm{Akt} / \mathrm{m}$ Tor activation regulates imatinib resistance development. Leukemia 19: 1774-1782, 2005.

21. Grunwald V, DeGraffenried L, Russel D, Friedrichs WE, Ray RB and Hidalgo $\mathrm{M}$ : Inhibitors of $\mathrm{mTOR}$ reverse doxorubicin resistance conferred by PTEN status in prostate cancer cells. Cancer Res 62: 6141-6145, 2002.

22. Mabuchi S, Altomare DA, Connolly DC, et al: RAD001 (Everolimus) delays tumor onset and progression in a transgenic mouse model of ovarian cancer. Cancer Res 67: 2408-2413, 2007.
23. Mabuchi S, Altomare DA, Cheung M, et al: RAD001 inhibits human ovarian cancer cell proliferation, enhances cisplatininduced apoptosis, and prolongs survival in an ovarian cancer model. Clin Cancer Res 13: 4261-4270, 2007.

24. Beuvink I, Boulay A, Fumagalli S, et al: The mTOR inhibitor RAD001 sensitizes tumor cells to DNA-damaged induced apoptosis through inhibition of p21 translation. Cell 120: 747-759, 2005 .

25. Shi Y, Frankel A, Radvanyi LG, Penn LZ, Miller RG and Mills GB: Rapamycin enhances apoptosis and increases sensitivity to cisplatin in vitro. Cancer Res 55: 1982-1988, 1995.

26. Wu C, Wangpaichitr M, Feun L, et al: Overcoming cisplatin resistance by mTOR inhibitor in lung cancer. Mol Cancer 4: 25$34,2005$.

27. Stordal B and Davey $M$ : Understanding cisplatin resistance using cellular models. IUBMB Life 59: 696-699, 2007.

28. Moore SM, Rintoul RC, Walker TR, Chilvers ER, Haslett C and Sethi T: The presence of a constitutively active phosphoinositide 3-kinase in small cell lung cancer cells mediates anchorageindependent proliferation via a protein kinase B and p70s6kdependent pathway. Cancer Res 58: 5239-5247, 1998.

29. Kamata S, Kishimoto T, Kobayashi S, Miyazaki M and Ishikura $\mathrm{H}$ : Possible involvement of persistent activity of the mammalian target of rapamycin pathway in the cisplatin resistance of AFP-producing gastric cancer cells. Cancer Biol Ther 6, 2007.

30. Liu LZ, Zhou XD, Qian G, Shi X, Fang J and Jiang BH: AKT1 amplification regulates cisplatin resistance in human lung cancer cells through the mammalian target of rapamycin/p70S6K1 pathway. Cancer Res 67: 6325-6332, 2007.

31. Dennis PB, Pullen N, Pearson RB, Kozma SC and Thomas G: Phosphorylation sites in the autoinhibitory domain participate in p70(s6k) activation loop phosphorylation. J Biol Chem 273 14845-14852, 1998

32. Pearson RB, Dennis PB, Han JW, et al: The principal target of rapamycin-induced p70s6k inactivation is a novel phosphorylation site within a conserved hydrophobic domain. EMBO J 14: 5279-5287, 1995.

33. Boulares AH, Yakovlev AG, Ivanova V, et al: Role of poly(ADP-ribose) polymerase (PARP) cleavage in apoptosis. Caspase 3-resistant PARP mutant increases rates of apoptosis in transfected cells. J Biol Chem 274: 22932-22940, 1999.

34. Jefferies HB, Fumagalli S, Dennis PB, Reinhard C, Pearson RB and Thomas G: Rapamycin suppresses 5'TOP mRNA translation through inhibition of p70s6k. EMBO J 16: 3693-3704, 1997.

35. Meng Q, Xia C, Fang J, Rojanasakul Y and Jiang B-H: Role of $\mathrm{PI} 3 \mathrm{~K}$ and AKT specific isoforms in ovarian cancer cell migration, invasion and proliferation through the p70S6K1 pathway. Cell Signal 18: 2262-2271, 2006.

36. Houghton PJ and Huang S: mTOR as a target for cancer therapy. Curr Top Microbiol Immunol 279: 339-359, 2004.

37. Dufner A, Andjelkovic M, Burgering BMT, Hemmings BA and Thomas G: Protein kinase B localization and activation differentially affect S6 kinase 1 activity and eukaryotic translation initiation factor 4E-binding protein 1 phosphorylation. Mol Cell Biol 19: 4525-4534, 1999.

38. Shi Y, Hsu JH, Hu L, Gera J and Lichtenstein A: Signal pathways involved in activation of p70S6K and phosphorylation of 4E-BP1 following exposure of multiple myeloma tumor cells to interleukin-6. J Biological Chem 277: 15712-15720, 2002.

39. Lee DF, Kuo HP, Chen CT, et al: IKK beta suppression of TSC1 links inflammation and tumor angiogenesis via the mTOR pathway. Cell 130: 440-455, 2007.

40. Ikezoe $\mathrm{T}$, Nishioka $\mathrm{C}, \mathrm{B}$ andobashi $\mathrm{K}$, et al: Longitudinal inhibition of PI3K/Akt/mTOR signaling by LY294002 and rapamycin induces growth arrest of adult T-cell leukemia cells. Leuk Res 31: 673-682, 2007.

41. Gervais M, Dugourd C, Muller L, et al: Akt down-regulates ERK1/2 nuclear localization and angiotensin II-induced cell proliferation through PEA-15. Mol Biol Cell 17: 3940-3951, 2006.

42. Basu A and Tu H: Activation of ERK during DNA damageinduced apoptosis involves protein kinase Cdelta. Biochem Biophys Res Commun 334: 1068-1073, 2005. 